\title{
Drosophila Ndfip is a novel regulator of Notch signaling
}

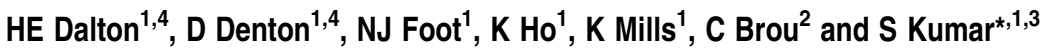

In the Drosophila wing, the Nedd4 ubiquitin ligases (E3s), dNedd4 and Su(dx), are important negative regulators of Notch signaling; they ubiquitinate Notch, promoting its endocytosis and turnover. Here, we show that Drosophila Nedd4 family interacting protein (dNdfip) interacts with the Drosophila Nedd4-like E3s. dNdfip expression dramatically enhances dNedd4 and $\mathrm{Su}(\mathrm{dx})$-mediated wing phenotypes and further disrupts Notch signaling. dNdfip colocalizes with Notch in wing imaginal discs and with the late endosomal marker Rab7 in cultured cells. In addition, dNdfip expression in the wing leads to ectopic Notch signaling. Supporting this, expression of dNdfip suppressed $\mathrm{Notch}^{+/}$wing phenotype and knockdown of dNdfip enhanced the Notch $^{+/}$wing phenotype. The increase in Notch activity by dNdfip is ligand independent as dNdfip expression also suppressed deltex RNAi and Serrate ${ }^{+/}$wing phenotypes. The opposing effects of dNdfip expression on Notch signaling and its late endosomal localization support a model whereby dNdfip promotes localization of Notch to the limiting membrane of late endosomes allowing for activation, similar to the model previously shown with ectopic Deltex expression. When dNedd4 or $\mathrm{Su}(\mathrm{dx})$ are also present, dNdfip promotes their activity in Notch ubiquitination and internalization to the lysosomal lumen for degradation.

Cell Death and Differentiation (2011) 18, 1150-1160; doi:10.1038/cdd.2010.130; published online 22 October 2010

Notch signaling is important in many cell-fate decisions, including cell differentiation and cell death, and its misregulation has been linked to cancer and developmental disorders. ${ }^{1-3}$ Notch receptor turnover in the absence of ligand is an important mechanism to prevent inappropriate signaling in unstimulated cells, requiring AP-2, AP-3 and HOPSdependent endocytic steps. ${ }^{4,5}$ Ubiquitination has a key role in regulating the endocytosis and subsequent downregulation of Notch. ${ }^{6}$ In the developing Drosophila wing, members of the Nedd4 family of HECT domain ubiquitin ligases (E3s), dNedd4 and Suppressor of deltex (Su(dx)), ubiquitinate and promote the endocytosis and turnover of Notch in a ligandindependent manner. Deltex (Dx) is a ring finger E3 known to positively regulate Notch signaling, with $d x$ mutants displaying loss of Notch phenotypes, such as distal wing blade notching. ${ }^{7}$ Dx overexpression can activate Notch in a ligand-independent manner, ${ }^{8}$ relying upon the endocytic pathway to traffic Notch to the limiting membrane of the lysosome, permitting $\gamma$-secretase cleavage and non-canonical activation of Notch. ${ }^{5}$ The activity of dNedd4 and $\mathrm{Su}(\mathrm{dx})$ overcome the ligandindependent activation of Notch by Dx by promoting the trafficking of Notch to the lysosomal lumen rather than the limiting membrane as promoted by Dx alone..$^{5,9,10}$

The Nedd 4 family are members of the HECT class of E3s, characterized by two to four WW domains (protein-protein interaction domains) and an $\mathrm{N}$-terminal $\mathrm{Ca}^{2+} /$ lipid binding $\mathrm{C} 2$ domain. ${ }^{11,12}$ Although many substrates directly bind the WW domains in Nedd4 E3 by means of PY or similar motifs, others rely on accessory/adapter proteins to promote ubiquitination. ${ }^{13}$ Two such proteins, Ndfip1 and Ndfip2, were initially identified as Nedd4 WW domain-binding proteins. ${ }^{14}$ Both Ndfip1 and 2 are ubiquitously expressed proteins with three transmembrane domains, and interact with several Nedd4 family members through PY motifs. ${ }^{15-17}$ Ndfip1 and 2 localize to Golgi, late endosomes and other vesicles and function as adapters and activators to facilitate the ubiquitination and regulation of a number of substrates. ${ }^{15,17-21}$ Here, we report on the Drosophila Nedd4 family interacting protein (dNdfip) homolog, and reveal that it is a novel regulator of Notch signaling.

\section{Results}

CG32177 encodes Drosophila Ndfip. Sequence comparison using Ndfip1 and Ndfip2 indicate that CG32177 encodes the only likely Ndfip homolog in Drosophila. ${ }^{15}$ Similar to Ndfip1 and Ndfip2, dNdfip contains an N-terminal portion that harbors three proline-rich motifs indicating a potential for binding WW-domain proteins, three transmembrane domains and a short C-terminal tail (Figure 1a). ${ }^{15}$ Throughout Drosophila development dNdfip is expressed at low levels (both RNA and protein) with highest levels observed from pupariation through to adulthood (data not shown). Like its mammalian counterparts, ${ }^{15,17}$ dNdfip showed significant colocalization with Rab7 in late

${ }^{1}$ Division of Haematology, Centre for Cancer Biology, SA Pathology, Adelaide, SA, Australia; ${ }^{2}$ Unité de Signalisation Moléculaire et Activation Cellulaire, URA 2582, CNRS, Institut Pasteur, Paris, France and ${ }^{3}$ Department of Medicine, University of Adelaide, North Terrace, Adelaide, SA, Australia

${ }^{*}$ Corresponding author: S Kumar, Division of Haematology, Centre for Cancer Biology, SA Pathology, Frome Road, Adelaide, SA 5000, Australia.

E-mail: sharad.kumar@health.sa.gov.au

${ }^{4}$ These authors contributed equally to this work.

Keywords: Nedd4; ubiquitination; Ndfips

Abbreviations: Dx, deltex; Ser, Serrate; Su(dx), Suppressor of deltex; Wg, wingless; Ndfip, Nedd4 family interacting protein; dNdfip, Drosophila Ndfip

Received 17.6.10; revised 16.9.10; accepted 21.9.10; Edited by E Baehrecke; published online 22.10.10 
a

PY1 PY2 PY3

TM1 TM2

Ndfip1/2

PY1

PY2 PY3

TM1 TM2

TM3

Drosophila Ndfip : PY1 = PPPY ; PY2 = LPTY ; PY3 = LPPPP

Mammalian Ndfip : PY1 = PPPY $;$ PY2 = PPSY $;$ PY3 = LPSY

b
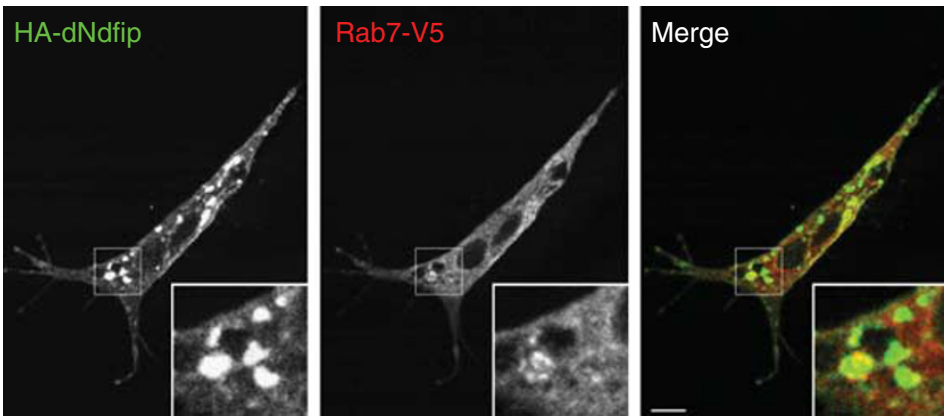

C
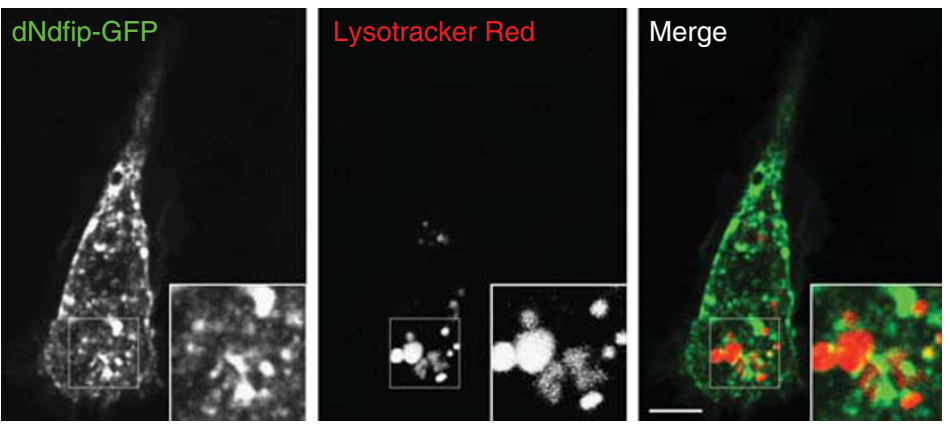

d
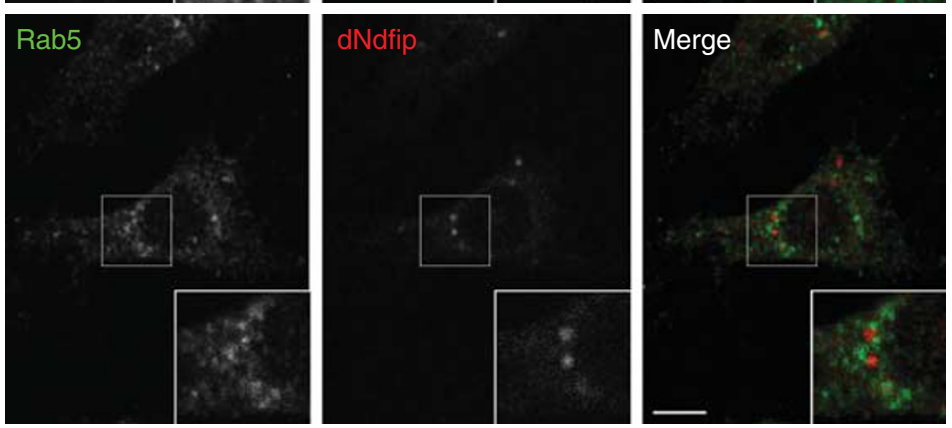

e
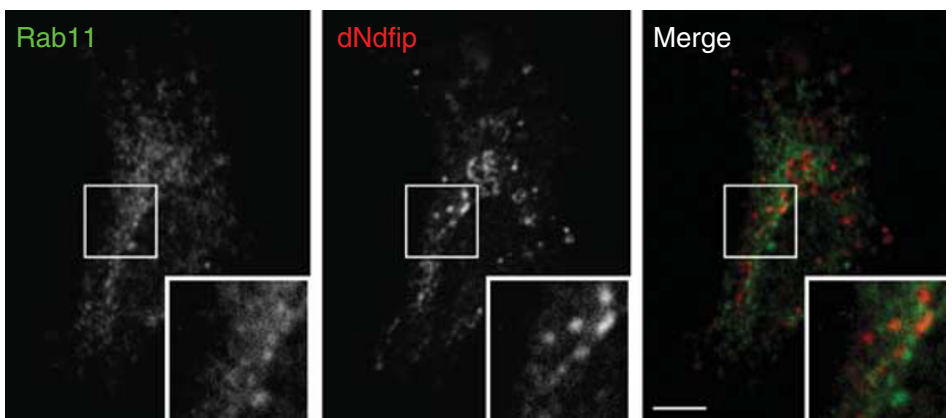

Figure 1 dNdfip localizes to the endosomal compartment, including the late endosome. (a) Schematic of Ndfip proteins showing the locations of PY motifs and transmembrane domains. PY motif sequences are also indicated. (b) Formaldehyde-fixed SL2 cells stained for HA-tagged dNdfip and V5-tagged late endosomal marker Rab7. (c) Live SL2 cells with GFP-tagged dNdfip (green) and the lysosome marker Lysostracker Red DND-99 (red). (d) Formaldehyde-fixed SL2 cells stained for dNdfip and early endosomal marker Rab5. (e) Formaldehyde-fixed SL2 cells stained for dNdfip and recycling endosomal marker Rab11. Insets are a $\times 2$ magnification of the corresponding box indicated. Scale bars $=5 \mu \mathrm{m}$ 
endosomes in Drosophila SL2 cells (Figure 1b, see inset), but not with Rab5 in early endosomes or Rab11 in recycling endosomes (Figure 1d and e). It does not appear to localize to lysosomes, though dNdfip-positive vesicles lie in close apposition to lysosomes (Figure 1c, see inset).

dNdfip interacts with all three Drosophila Nedd4 family E3 ubiquitin ligases. As mammalian Ndfips interact with multiple E3s, we tested whether dNdfip binds the Drosophila Nedd4 family E3s dNedd4, Su(dx) and dSmurf. Tagged proteins were expressed in SL2 cells and subjected to coimmunoprecipitation. dNdfip coimmunoprecipitated with all three E3s (Figure 2a-c) and this interaction was mediated by the three proline-rich motifs, as mutants of each PY motif showed some loss of dNedd4 binding (compared with wild-type dNdfip, normalized to 1.0), and the triple PY mutant showed the least binding to dNedd4 (last lane, only 0.2 relative to wild type, Figure $2 d$ ). Further, dNdfip partially colocalized with all three E3s in a punctate endosomal-like manner in SL2 cells (Figure 3a-c). In mammalian cells, Ndfips appear to alter the subcellular location of E3s, with Ndfip2 promoting colocalization in late endosomes of Nedd4, Itch and Smurf2. ${ }^{17,19}$ Also, Ndfip1 promotes the localization of Nedd4, Nedd4-2 and Itch to exosomes that arise from a late endosomal origin. ${ }^{22}$ dNdfip expression, however, did not significantly alter the localization of the E3s (Figure 3). Furthermore, similar to mammalian Ndfips, ${ }^{15,17}$ dNdfip is ubiquitinated by Nedd4 E3s, such as dNedd4 and Su(dx)
(Supplementary Figure S1). These observations are consistent with a predicted function for dNdfip as an adapter/regulator of E3s.

dNdfip augments the function of $d N e d d 4$ and $S u(d x)$ in regulating Notch signaling. During Drosophila wing development, expression of $\mathrm{dNedd} 4$ and $\mathrm{Su}(\mathrm{dx})$ reduces Notch signaling, producing a notched wing phenotype. ${ }^{9,10}$ In order to investigate whether dNdfip has a role with dNedd4 and $\mathrm{Su}(\mathrm{dx})$ in regulating Notch signaling, we examined the overexpression of dNdfip as no specific mutant alleles were available. Overexpression of dNedd4 along the anterior-posterior boundary of the wing using the patchedGAL4 (ptc-GAL4) driver resulted in a disrupted wing with thick veins and loss of the adult wing margin, consistent with its known role in downregulation of Notch signaling (Figure 4b). ${ }^{9,10}$ Co-expression with dNdfip enhanced the dNedd4 wing phenotype, with severe loss of the wing margin producing smaller wings (Figure 4e, compared with Figure 4b). Overexpression of $\mathrm{Su}(\mathrm{dx})$ using ptc-GAL4 produced a mild disruption of wing veins, a phenotype consistent with Notch downregulation (Figure 4c). Co-expression with dNdfip enhanced the Su(dx) wing phenotype, with loss of the adult wing margin (Figure 4f, compared with Figure 4c).

Because of significant mortality in the progeny expressing both E3 and dNdfip using the ptc-GAL4 driver, we utilized the wing-specific driver MS1096-GAL4 to further investigate the genetic interaction between $d N d f i p$ and $d N e d d 4$ or $S u(d x)$.

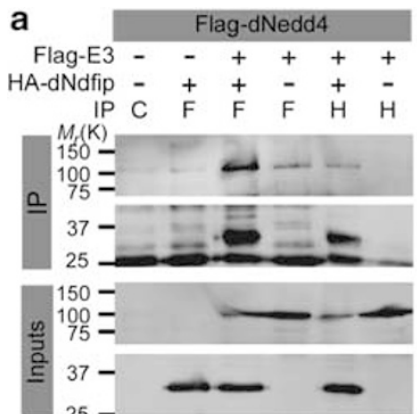

b

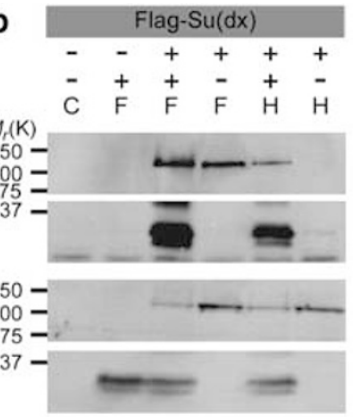

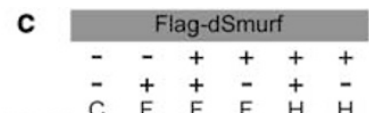

$\begin{array}{llllll}- & + & + & - & + & - \\ \mathrm{C} & \stackrel{\mathrm{F}}{\mathrm{F}} & \overrightarrow{\mathrm{F}} & \stackrel{\mathrm{H}}{\mathrm{H}}\end{array}$

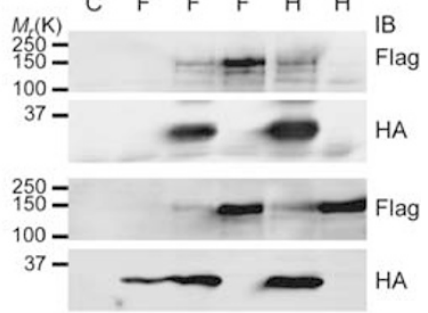

d Flag-dNedd4 -++++++++

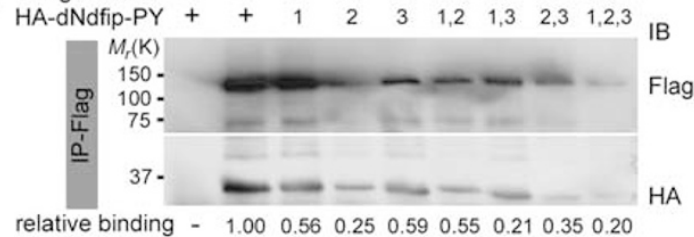

relative binding $-\begin{array}{llllllll}1.00 & 0.56 & 0.25 & 0.59 & 0.55 & 0.21 & 0.35 & 0.20\end{array}$

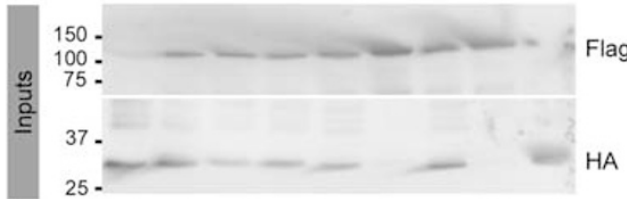

Figure 2 dNdfip interacts with the Drosophila E3s dNedd4, Su(dx) and dSmurf in a PY-dependent manner. (a-c) Lysates from SL2 cells co-transfected with wild-type HA-tagged dNdfip and Flag-tagged E3 ligases (a, dNedd4; b, Su(dx); c, dSmurf) were subjected to immunoprecipitation with $5 \mu$ g of control (equal mix of anti-Flag and anti-HA), anti-Flag or anti-HA antibodies as indicated (C, F and H, respectively). Input controls were $5 \%$ of each protein lysate. Proteins were separated by SDS-PAGE and immunoblotted for Flag or HA. (d) SL2 cells were co-transfected with wild-type Flag-tagged dNedd4 and HA-tagged dNdfip (wild type ( + ) or PY mutants as indicated). Immunoprecipitations were carried out as in (a-c). Relative binding indicates the normalized protein levels of dNdfip binding to dNedd4 

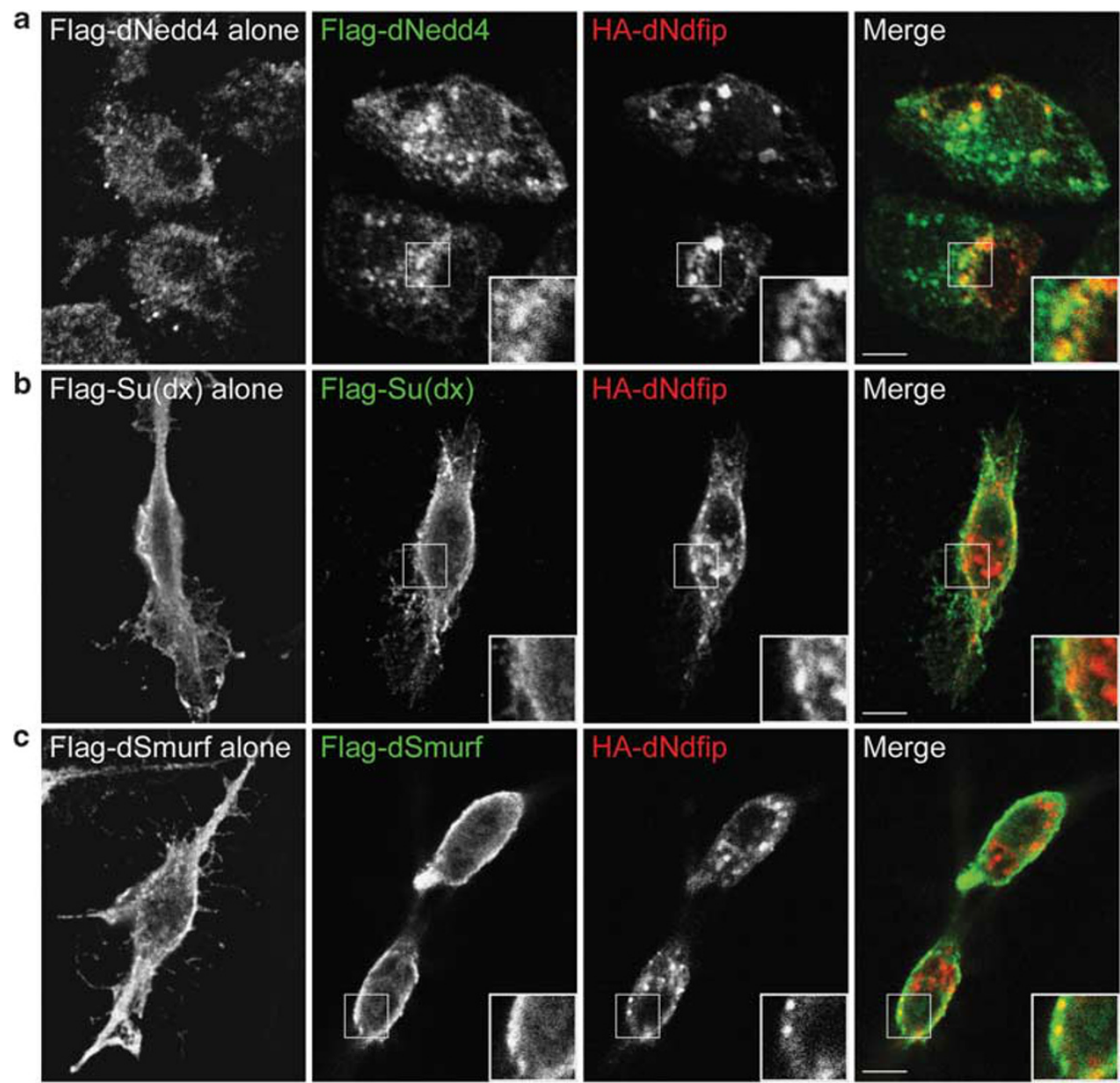

Figure 3 dNdfip partially colocalizes with the Drosophila E3s dNedd4, Su(dx) and dSmurf. (a-c) Transfected SL2 cells were fixed and stained for Flag-tagged E3 ligases (a, dNedd4; $\mathbf{b}, \mathrm{Su}(\mathrm{dx}) ; \mathbf{c}, \mathrm{dSmurf}$ ) alone (left hand side panel) or with HA-tagged dNdfip (other panels). Insets are $\times 2$ magnifications of the marked boxes that highlight points of colocalization, see right hand side merge panels. Scale bars $=5 \mu \mathrm{m}$

Overexpression of dNedd4 produced a severely disrupted wing (Figure $5 \mathrm{~b}$ ), with thick veins and a loss of bristles at the wing margin, consistent with its known role in downregulation of Notch signaling. ${ }^{9,10}$ Co-expression with dNdfip resulted in an enhanced wing phenotype, with little intervein space evident, thick veins and smaller wings (Figure 5e, compared with Figure 5b). Overexpression of Su(dx) using MS1096GAL4 produced a mild phenotype consistent with Notch downregulation, with vein thickening especially near the wing margins (Figure 5c). As expected, co-expression with dNdfip resulted in a severely disrupted wing, with increased vein thickening and a smaller wing size (Figure $5 f$, compared with Figure 5c). The enhancement of the Nedd4 and Su(dx) wing phenotypes by dNdfip indicates that dNdfip is functioning to augment E3 function.

The Notch-like wing phenotypes caused by the expression of $\mathrm{dNedd} 4$ and $\mathrm{Su}(\mathrm{dx})$ are due in part to the defective induction of the Notch target gene, wingless $(w g)$, in the dorso-ventral organizer of the wing imaginal disc. ${ }^{9,10}$ Thus, dNdfip may also be involved in downregulating Notch signaling. To confirm that the disrupted wing phenotypes are due at least in part to disruption of Notch signaling, we examined Wg staining in the developing wing. Because of the secreted nature of $\mathrm{Wg}$ and to rule out any direct role on $\mathrm{Wg}$ protein turnover, we also examined Cut, an established marker of Notch activity. 23,24 Similar to $\mathrm{Wg}$, high levels of Notch activity induces Cut expression along the dorso-ventral organizer. ${ }^{23,24}$ Expression of dNedd4 along the anterior-posterior boundary (ptc-GAL4) resulted in a reduction of $\mathrm{Wg}$ and Cut at the dorso-ventral region (Figure $4 \mathrm{~h}$ and $\mathrm{n}$; compared with wild-type Figure $4 \mathrm{~g}$ and $\mathrm{m}$ ) and this was broadened in the presence of dNdfip (Figure $4 \mathrm{k}$ and $\mathrm{q}$ ). Su(dx) did not appear to disrupt Wg and Cut expression (Figure 4i and o), whereas coexpresion of dNdfip resulted in a disruption to $\mathrm{Wg}$ and Cut at the central part of the dorso-ventral boundary (Figure $4 \mathrm{I}$ and $\mathrm{r}$ ). When dNedd4 was overexpressed along the dorso-ventral boundary (MS1096GAL4), Wg and Cut were present in the typical pattern; however, their expression was restricted to a thinner layer of cells and appeared more speckled than in wild type, consistent with a downregulation of Notch signaling (Figure $5 \mathrm{~h}$ and $\mathrm{n}$; compare with wild type Figure $5 \mathrm{~g}$ and $\mathrm{m}$ ). Co-expression of dNedd4 and dNdfip resulted in a loss of $\mathrm{Wg}$ and Cut altogether in the disc including the dorsal-ventral boundary (Figure $5 \mathrm{k}$ and $\mathrm{q}$ ). These wing discs were no longer similar to the wild type, but were quite undulated and wrinkled. MS1096-GAL4-driven expression of $\mathrm{Su}(\mathrm{dx})$ did not appear to 


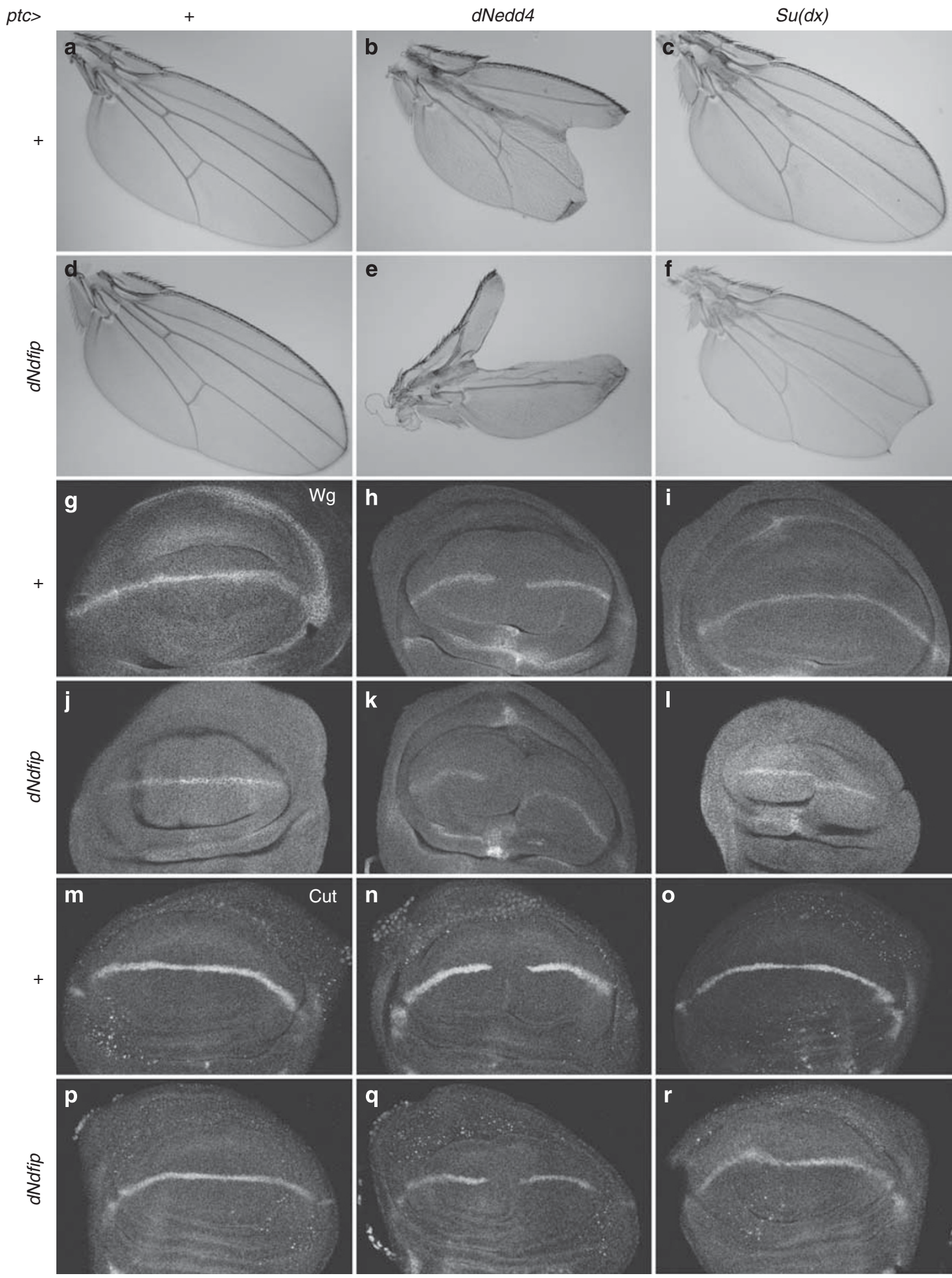

Figure $4 d N d f i p$ enhances wing phenotypes conferred by $d N e d d 4$ and $S u(d x)$. The ptc-GAL4 driver control (a, $\mathbf{g}$ and $\mathbf{m})$ and driving expression of $U A S-d N e d d 4 G S$ alone $(\mathbf{b}, \mathbf{h}$ and $\mathbf{n}), U A S-S u(d x)$ alone (c, $\mathbf{i}$ and $\mathbf{o}$ ) and UAS-HA-dNdfip alone (d, $\mathbf{j}$ and $\mathbf{p}$ ) and co-overexpression of UAS-dNedd4GS with UAS-HA-dNdfip (e, $\mathbf{k}$ and $\mathbf{q})$ and UAS-Su(dx) with UAS-HA-dNdfip (f, I and $\mathbf{r}$ ). (a-f) Adult wings from the indicated genetic combinations. ( $\mathbf{g}-\mathbf{I})$ Wing discs from third instar larvae fixed and stained for Wg as a marker of Notch activity. (m-r) Wing discs from third instar larvae fixed and stained for Cut as a marker of Notch activity. Scale bar $=100 \mu \mathrm{m}$

disrupt $\mathrm{Wg}$ or Cut expression along the dorso-ventral boundary (Figure $5 \mathrm{i}$ and $\mathrm{o}$ ). However, co-expression of $\mathrm{Su}(\mathrm{dx})$ with dNdfip resulted in a loss of $\mathrm{Wg}$ and Cut at the dorsal-ventral boundary and undulated discs (Figure $5 \mathrm{l}$ and $\mathrm{r}$ ). These data indicate that dNdfip enhances the ability of both dNedd4 and $\mathrm{Su}(\mathrm{dx})$ to downregulate Notch signaling. 
MS1096>
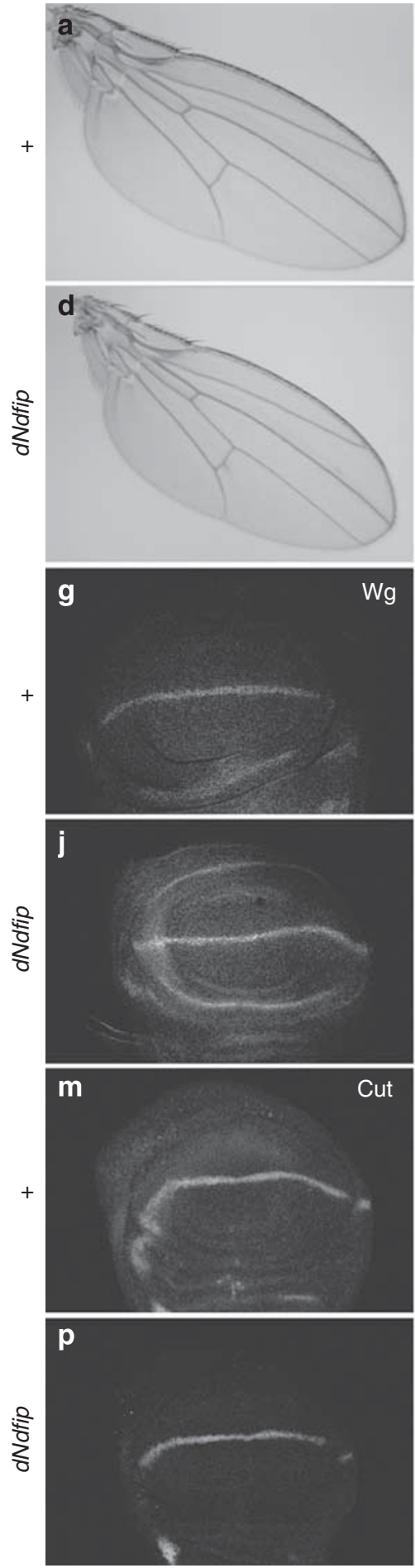

$d N e d d 4$

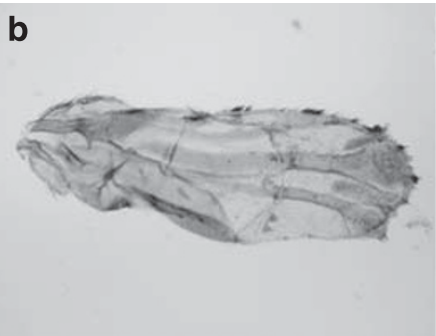

e

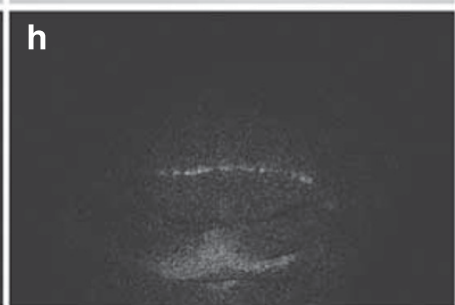

k

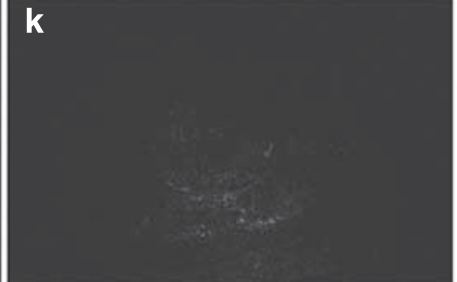

n

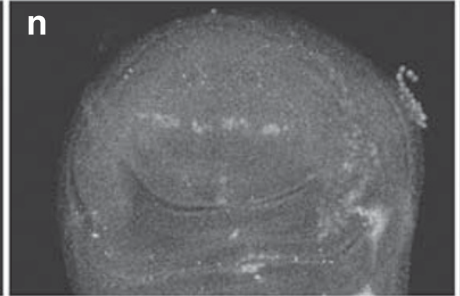

q

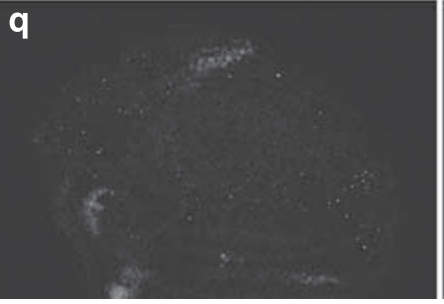

$S u(d x)$

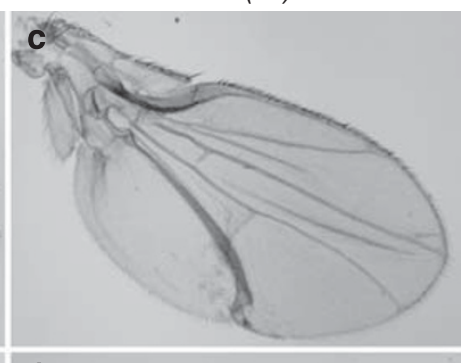

f
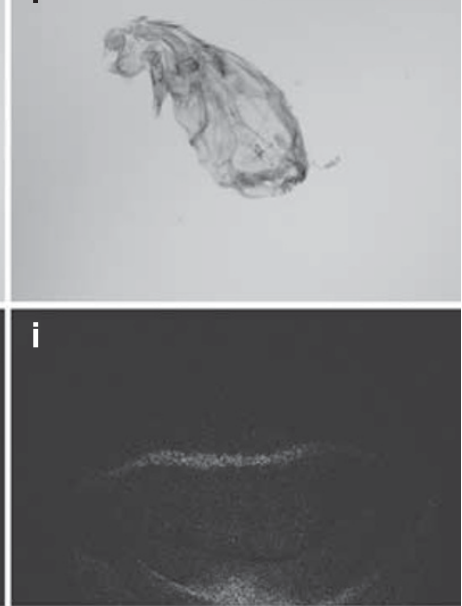

I

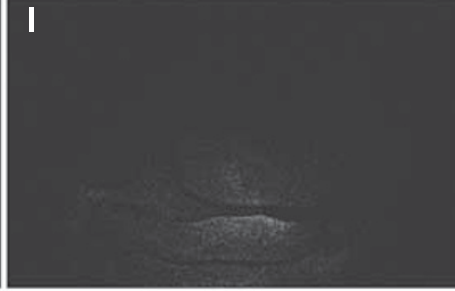

o

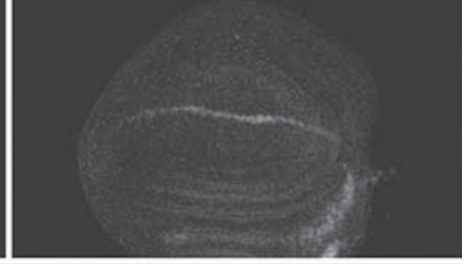

$r$

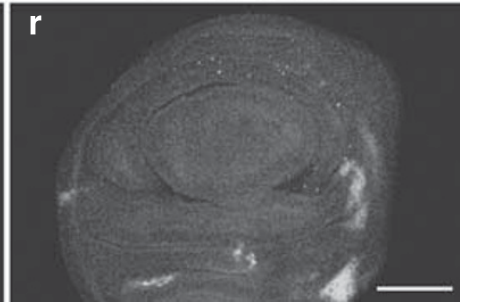

Figure 5 dNdfip enhances wing and wing disc phenotypes conferred by dNedd4 and Su(dx). The wing-specific driver MS1096-GAL4 control (a, $\mathbf{g}$ and $\mathbf{m})$ and with UAS-dNedd4GS alone (b, $\mathbf{h}$ and $\mathbf{n})$, UAS-SU(dx) alone (c, $\mathbf{i}$ and $\mathbf{0})$, UAS-HA-dNdfip alone (d, $\mathbf{j}$ and $\mathbf{p})$ and co-overexpression of UAS-dNedd4GS with UAS-HA-dNdfip $(\mathbf{e}, \mathbf{k}$ and $\mathbf{q})$ and $U A S-S u(d x)$ with UAS-HA-dNdfip (f, I and $\mathbf{r})$.(a-f) Adult wings from the indicated genetic combinations. (g-l) Wing discs from third instar larvae fixed and stained for Wg as a marker of Notch activity. ( $\mathbf{m}-\mathbf{r})$ Wing discs from third instar larvae fixed and stained for Cut as a marker of Notch activity. Scale bar $=100 \mu \mathrm{m}$

dNdfip genetically interacts with Notch, deltex and Serrate. Interestingly, although the overexpression of a single copy of dNdfip alone in the Drosophila wing (using MS1096-GAL4 or ptc-GAL4) has minimal effects on the adult wing (Figures $5 \mathrm{~d}$ and $6 \mathrm{a}$ ), high levels of dNdfip expression using two copies of the transgene resulted in severe wing disruption (Figure 6c), with increased bristles present at the wing margin consistent with Notch activation. Examination of 

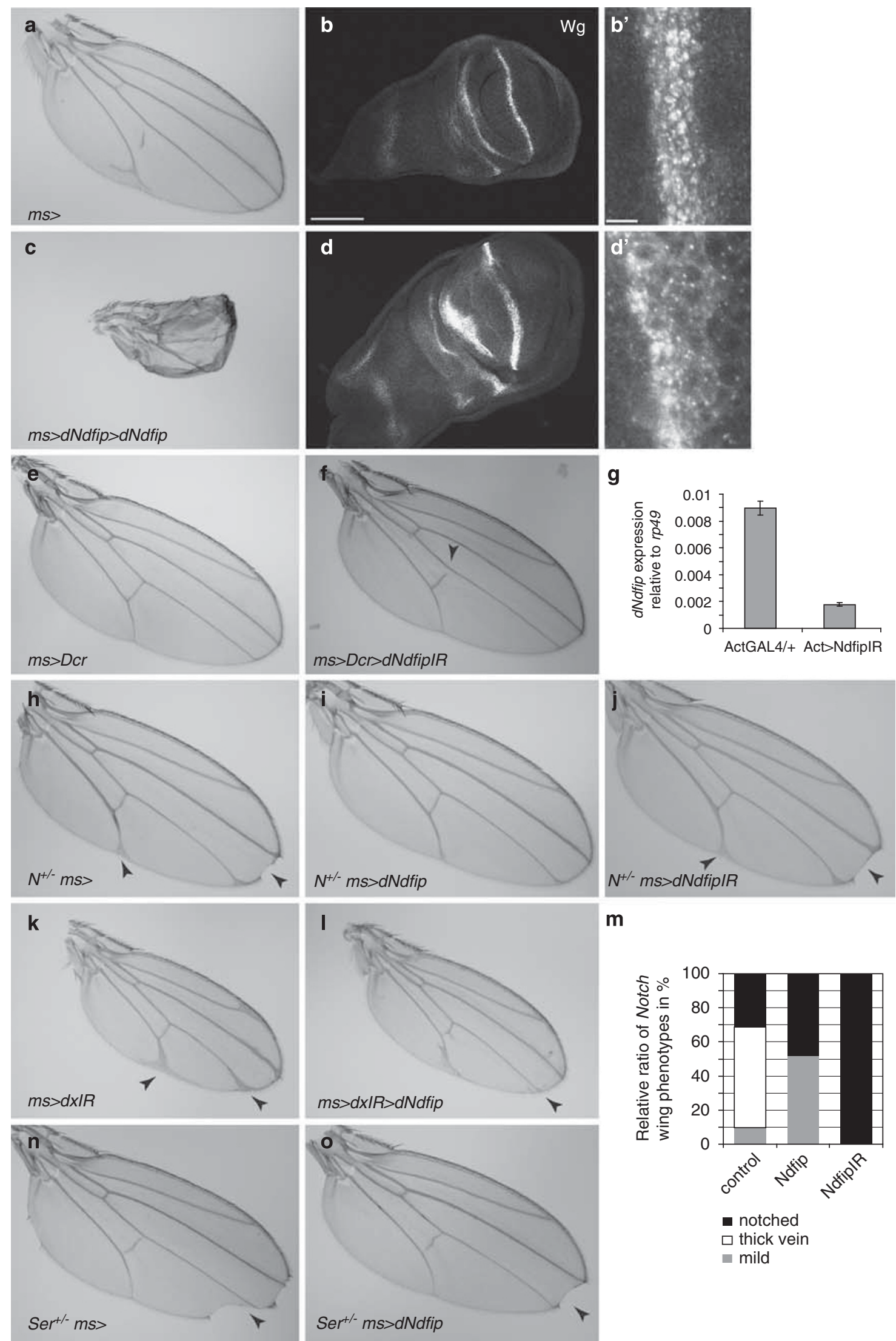
Wg expression revealed modest effects on Wg with one copy of dNdfip, however, two copies resulted in broadening of $\mathrm{Wg}$ at the dorso-ventral boundary, as well as increased Wg more dorsally, in the disc (Figure 6, compare Figure 6b and $b^{\prime}$ with Figure $6 \mathrm{~d}$ and $\mathrm{d}^{\prime}$ ). The increase in $\mathrm{Wg}$ levels was verified using the enhancer trap line $w g$-lac $Z$, which also showed increased $\beta$-galactosidase levels (data not shown). This increase in Notch signaling was surprising given that dNdfip when co-expressed with $\mathrm{dNedd} 4$ and $\mathrm{Su}(\mathrm{dx})$ was functioning to further reduce $\mathrm{Wg}$.

To further investigate the contribution of dNdfip to Notch signaling, dNdfip was overexpressed or knocked down in a Notch allele, $N^{264-39}$. Heterozygous $N^{264-39 /+}$ adult wings have a characteristic mild notched wing margin phenotype (Figure 6h). Expression of dNdfip in $N^{264-39 /+}$ mutant wings suppressed the wing margin notching, bristle loss and slight vein thickening near the wing blades (Figure 6, compare Figure $6 \mathrm{~h}$ with Figure $6 \mathrm{i}$ ). To examine the effect of reduced dNdfip on the $\mathrm{N}^{264-39 /+}$ mutant phenotype, RNAi was used to target $d N d f i p$ in the wing using MS1096-GAL4. This line shows $\sim 80 \%$ reduction in $d N d f i p$ expression, as assessed by real-time PCR (Figure 6g). Knockdown of $d$ Ndfip in the wing resulted in minor vein defects (Figure 6, compare Figure $6 f$ with Figure 6e). Furthermore, the knockdown of dNdfip in $N^{264-39 /+}$ mutant wings enhanced the wing margin notching and slight vein thickening near the wing blades (Figure 6, compare Figure 6h with Figure 6j).

These opposing regulatory roles for dNdfip are reminiscent of Dx phenotypes, which when ectopically expressed leads to non-canonical (ligand-independent) activation of Notch, a phenotype that can be overcome by co-expression of $\mathrm{Su}(\mathrm{dx})$ or dNedd4 or by loss of members of the endocytic pathway, such as AP-3 and HOPS complex members. ${ }^{5,9,10}$ Loss of $d x$ results in a loss of Notch signaling, ${ }^{7}$ and using RNAi we confirmed that reducing $d x$ in the developing wing results in minor vein thickening phenotypes near the wing margins (Figure 6k). Overexpression of dNdfip suppressed the $d x$ knockdown phenotypes, with a reduction of the vein thickening phenotypes (Figure 6, compare Figure 6k with Figure 6l). This suggests that dNdfip can activate Notch in the absence of Dx. Notch signaling is activated by binding of the ligands Serrate (Ser) or Delta, that results in proteolytic processing of Notch, releasing the Notch intracellular domain, which then translocates to the nucleus to regulate gene expression. ${ }^{25}$ Similar to the Notch mutant phenotype, heterozygous Ser ${ }^{1}$ wings have a notched phenotype. The Ser ${ }^{1 /+}$ wing phenotype is suppressed by the overexpression of dNdfip (Figure $6 n$ and o). This suggests that dNdfip expression increases Notch activity, and this can occur independent of ligand activation.
In addition, dNdfip colocalizes with Notch in the wing imaginal disc (Figure 7a-f) and in $\mathrm{S} 2$ cells stably expressing Notch (data not shown) supporting the role of dNdfip in regulating Notch signaling. Staining of endogenous dNdfip revealed broad punctate expression across the wing disc with accumulation at the margins adjacent to $\mathrm{Wg}$ expression at the dorso-ventral boundary, particularly in the anterior compartment, and a lack of expression where $\mathrm{Wg}$ is expressed (Figure $\left.7 g-i^{\prime}\right)$. This observation supports a role for dNdfip in refining the boundary of Notch-responsive cells.

\section{Discussion}

Our data suggest that dNdfip is a functional homolog of mammalian Ndfip1 and Ndfip2. dNdfip interacts and colocalizes with all three Drosophila Nedd4 family E3 ubiquitin ligases. Moreover, dNdfip enhanced the wing phenotypes of $d N e d d 4$ and $S u(d x)$, and further downregulated Wg expression in wing discs, indicating a negative regulatory role for dNdfip in Notch signaling. The knockdown of dNdfip did not show any modification of the dNedd4 wing phenotype (data not shown). This may be due to several reasons, such as the strong phenotype of dNedd4 overexpression, incomplete knockdown of $d N d f i p$ or the involvement of dNedd4 in the regulation of other pathways.

It has been reported that $\mathrm{Su}(\mathrm{dx})$ and Notch interact directly through a PY motif in the Notch receptor. ${ }^{10}$ This interaction is mediated by WW domain 4 of $\mathrm{Su}(\mathrm{dx})$, which lies in tandem with WW domain 3. Furthermore when unbound, WW domain 3 inhibits the interaction between Notch PY and WW domain 4 thus, if WW domain 3 is occupied by another factor, Su(dx) is better able to bind Notch. ${ }^{26}$ As all three PY-like motifs in dNdfip must be mutated to disrupt binding to dNedd4, and as these motifs are known to bind WW domains, it is likely that dNdfip is capable of binding multiple WW domains in dNedd4 and $\mathrm{Su}(\mathrm{dx})$ and thus, may serve as a suitable candidate to overcome the inhibitory influence of WW domain 3 in binding Notch by $\mathrm{Su}(\mathrm{dx})$. Also, most mammalian isoforms of Notch lack a PY motif and, therefore, depend upon known adapters to mediate the interaction with E3s, such as Itch $(\mathrm{Su}(\mathrm{dx})$ ortholog). ${ }^{27,28}$ Recently, however, Itch-mediated turnover of Notch1 receptor in the absence of ligand has been shown to depend upon a bridging factor that is not Numb, nor Dx, nor the adapters $\beta$-arrestin 1 or $2 .^{28}$ It is possible that Ndfips serve in an adapter role in Notch regulation. dNdfip may facilitate E3 access to Notch, as it interacts with both dNedd4 and $\mathrm{Su}(\mathrm{dx})$ and colocalizes with Notch in wing discs and in S2 cells stably

Figure 6 Expression of dNdfip in the wing disrupts wing structure, increases Wg expression and modifies Notch-dependent wing phenotypes. (a-d) MS1096-GAL4 driver control $\left(\mathbf{a}-\mathbf{b}^{\prime}\right)$ and with two-copies UAS-HA-dNdfip (c- $\left.\mathbf{d}^{\prime}\right)$ showing adult wing phenotypes (a and $\left.\mathbf{c}\right)$ and $\mathbf{W g}$ expression in third instar larval wing discs as a measure of Notch activation $\left(\mathbf{b}, \mathbf{b}^{\prime}, \mathbf{d}\right.$ and $\left.\mathbf{d}^{\prime}\right)$. Scale Bars $=100 \mu \mathrm{m}(\mathbf{b}) ; 5 \mu \mathrm{m}\left(\mathbf{b}^{\prime}\right)$. $\mathbf{b}$ And $\mathbf{d}$ are maximum pixel intensity merges of six z-stack images each, $2.0 \mu \mathrm{m}$ apart. $\mathbf{b}^{\prime}$ And $\mathbf{d}^{\prime}$ are merges with maximum pixel intensity of four z-stack images each, $1.0 \mu \mathrm{m}$ apart. (e and f) Adult wings from MS1096-GAL4 UAS-Dcr driver control (e) and with UAS-dNdfip/R (f). To improve the knockdown efficiency of $d N d f i p$ by RNAi Dicer (Dcr) was co-expressed. (g) Real-time PCR analysis of the knock down of $d N d f i p$ in Act5c-GAL4UAS-dNdfip/Rflies. (h-j) Adult wings from $N^{264-39 /+}$ flies carrying the MS1096-GAL4 driver control (h) with UAS-HA-dNdfip (i) and with UAS-dNdfip/R (j). (k and I) Adult wings from flies carrying the MS1096-GAL4 driver and UAS-deltexIR RNAi (k) and with UAS-HA-dNdfip (I). (m) Quantification of the variation in the Notch wing phenotypes observed. The phenotypes were classed as mild with mostly normal wings but having minor vein defects, thick vein (arrow in $\mathbf{h}$ ) at margin without wing notching and notched (arrow in $\mathbf{j}$ ) with wing margin notching and vein thickening. (n and $\mathbf{0})$ Adult wings from Ser ${ }^{1 /+}$ flies with MS1096-GAL4 driver control (n) and with UAS-HA-dNdfip (o). Arrows indicate various wing defects 

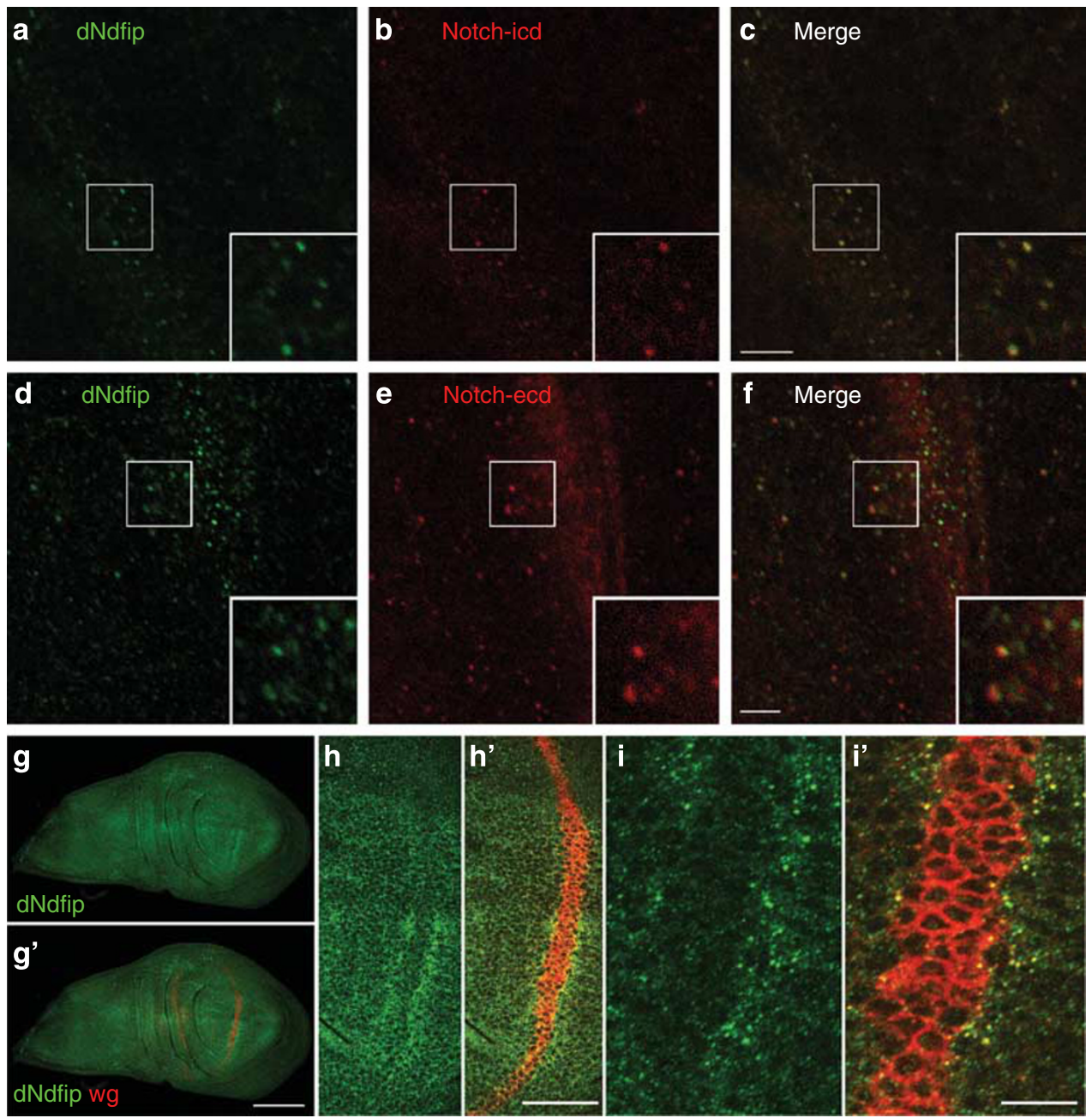

Figure 7 Endogenous dNdfip colocalizes with Notch and apposes Wg at the dorso-ventral wing boundary. (a-c) Wild-type third instar larval wing discs stained for dNdfip (a, c; green) and Notch intracellular domain (b, c; red, Notch-icd). (d and e) Wild-type third instar larval wing discs stained for dNdfip (d, f; green) and Notch extracellular domain (e, $\mathbf{f}$; red, Notch-ecd). Images are a maximum pixel intensity merge of four $z$-stack images each, $0.10-\mu \mathrm{m}$ apart. Insets are $\times 2$ magnifications of the boxes indicated, colocalization of Notch and dNdfip can be seen as yellow puncta in inset box c. Scale bar $=5 \mu \mathrm{m}$. (g-i $\left.\mathbf{i}^{\prime}\right)$ Wild-type third instar larval wing discs stained for dNdfip ( $\mathbf{g}, \mathbf{h}, \mathbf{i} ;$ green) and $\mathbf{W g}\left(\mathbf{g}^{\prime}, \mathbf{h}^{\prime}, \mathbf{i}^{\prime} ;\right.$ red). $\mathbf{h}, \mathbf{h}^{\prime}, \mathbf{i}$ and $\mathbf{i}^{\prime}$ are a maximum pixel intensity merge of five $\mathbf{z}$-stack images, $0.500 \mu \mathrm{m}$ apart. Scale bars $=100 \mu \mathrm{m}\left(\mathbf{g}^{\prime}\right) ; 40 \mu \mathrm{m}\left(\mathbf{h}^{\prime}\right) ; 10 \mu \mathrm{m}\left(\mathbf{i}^{\prime}\right)$

expressing Notch. ${ }^{29}$ Indeed we have observed such a role for dNdfip in the promotion of ubiquitination of Malvolio, ${ }^{30}$ the ortholog of the known Ndfip-regulated iron transporter DMT1 (our unpublished observations). ${ }^{20}$

Another possible regulatory mode of action for dNdfip may include the direct activation of Nedd4 family E3s, as has been observed for Ndfip2 that promotes the auto-activation of several E3s, including Itch. ${ }^{19}$ Furthermore, Ndfip2 promotes the ubiquitination of the PY-containing substrates $c$-Jun and JunB, as well as the non-PY-containing substrate Endophilin A1. ${ }^{19}$ In this model, multiple PY-WW domain interactions overcome an autoinhibitory state mediated by the intramolecular binding of the catalytic HECT domain and the N-terminal regions of Itch and Nedd4. dNdfip also mediates multiple PY-WW domain interactions and thus, may function in a similar fashion. Indeed dNdfip promotes the ubiquitination of dNedd4 in SL2 cells, however, it also promotes ubiquitination of dNedd4 catalytic mutants and thus, the activation may be in trans from abundant endogenous dNedd4 (our unpublished observations).
dNdfip colocalizes with Notch in endosomal-like puncta in the wing disc and in S2 cells stably expressing Notch. Expression of dNdfip in the Drosophila wing suppressed the wing notching phenotypes caused by the $N^{264-39}$ allele, whereas dNdfip knockdown resulted in an enhancement of the $N^{264-39}$ phenotype, suggestive of a positive role of dNdfip in Notch signaling. dNdfip expression also suppressed the loss-of-Notch-associated wing phenotypes conferred by the loss of $d x$ or Sersuggesting that dNdfip can activate Notch in a ligand-independent manner. The expression of endogenous dNdfip in the wing, with low expression in the Wg-expressing dorso-ventral boundary and higher punctate expression immediately adjacent to this boundary suggests that dNdfip may have a regulatory role in refining Notch signaling to define the dorso-ventral boundary. This positive regulation of Notch signaling appears contradictory to the previously discussed negative regulation. However, these opposing phenotypes observed for dNdfip expression, in the presence and absence of Nedd4 family E3s, are consistent with a model of Notch trafficking established for Dx-promoted Notch activation and 
regulation. ${ }^{5}$ Dx promotes Notch endocytosis in a HOPS- and AP-3-dependent manner. It promotes targeting of Notch to the limiting membranes of late endosomes and lysosomes. ${ }^{8}$ Once at the lysosome, the extracellular domain of Notch is degraded within the lysosomal lumen, then the Notch intracellular domain can be cleaved by presenilin $\gamma$-secretase, thereby activating Notch signaling in a non-canonical manner. ${ }^{5}$ When $\mathrm{dNedd} 4$ or $\mathrm{Su}(\mathrm{dx})$ are present, Notch is ubiquitinated and is trafficked to the lysosomal lumen, wherein it is degraded and signaling does not occur. ${ }^{5}$ We propose that dNdfip functions in a manner similar to Dx, in that alone it can promote Notch trafficking to the late endosome/lysosome and in the absence of E3, Notch remains at the limiting membrane, whereby it can be activated in a non-canonical manner. When E3s are present, dNdfip enhances the ability of the E3 to downregulate Notch signaling, promoting the E3-mediated ubiquitination and late endosomal trafficking of Notch cargo, wherein it is internalized and delivered to the lysosomal lumen for degradation. Unlike Dx, dNdfip greatly augments the ability of dNedd4 and $\mathrm{Su}(\mathrm{dx})$ to negatively regulate Notch signaling.

Although our data clearly suggest that dNdfip is a novel regulator of Notch signaling, the potent genetic interactions observed between dNdfip and both dNedd4 and Su(dx) are likely to be due to more than negative regulation of Notch signaling alone. The undulating wing discs and the resultant small wings point to perturbation of other signaling pathways. Indeed mammalian Ndfips have recently been shown to regulate members of the EGF pathway by promoting Nedd4 family E3-mediated ubiquitination of several pathway members, including PTEN, c-Cbl and Src family kinases. ${ }^{31}$ Further studies await availability of specific $d N d f i p$ mutants, as all currently available transposon insertion lines also affect Keren, a gene that overlaps with $d N d f i p$, sharing a common $5^{\prime}$-UTR and thus, complicating the analysis of the specific contribution of $d N d f i p$ to the phenotype.

\section{Materials and Methods}

Cloning and cell culture. The full-length clone of dNdfip (Genbank accession No. AAF49316) with and without an N-terminal HA-tag was cloned into pIE4 (Novagen) for cell culture expression and HA-dNdfip was cloned into pUAST ${ }^{32}$ for generation of transgenic flies. A C-terminal GFP-tagged dNdfip clone was also generated in plE4. Mutations of the PY motifs in pIE4-HA-dNdfip were generated by site-directed mutagenesis and corresponded to the following amino acid changes $(P Y 1=Y 27 A ; P Y 2=Y 86 A ; P Y 3=P 114 V, P 115 V)$. Full length wild type and catalytically inactive versions of the E3 ubiquitin ligases, dNedd4 (DGC clone SD04682, Genbank accession No. AY061595), Su(dx) (DGC clone LD32282, Genbank accession number BT021390) and dSmurf (Genbank accession number BT021410), were cloned with an N-terminal Flag tag into plE4. HA-tagged Ubiquitin was subcloned from pMT123 ${ }^{33}$ into plE4 for ubiquitination experiments. Rab7 was cloned into plB/V5-His (Invitrogen, Carlsbad, CA, USA) to generate a C-terminal V5 fusion construct for cell culture expression. Schneider L2 (SL2) cells were cultured and transiently transfected as described previously. ${ }^{34}$

Fly stocks, genetics and analyses. Transgenic UAS-HA-dNdfip flies were generated by standard $P$-element-mediated germline transformation. Multiple lines of flies with independent transgene insertion were established. Other fly lines used include MS1096-GAL4; ptc-GAL4; UAS-Dcr; $N^{264-39}$; and $S e r^{1}$ from the Bloomington Drosophila Stock Center. UAS-dNedd4GS; ${ }^{9}$ UAS-Su(dx), ${ }^{35}$ UAS-dNdfipIR and UAS-deltexIR from Vienna Drosophila RNAi Center ${ }^{36}$. Flies were maintained and experiments performed at $25^{\circ} \mathrm{C}$.

Adult wing analysis was performed by dehydrating adult flies in xylene before mounting wings in Canada Balsam. Wings were photographed using a microscope with a $4 \times$ UPlan FI objective (BX51; Olympus, Tokyo, Japan) and a camera (DP70;
Olympus) at room temperature. Images were captured with Olysia Bioreport software (Olympus) and compiled using Photoshop CS5 (Adobe, San Jose, CA, USA).

Antibody reagents. A glutathione-S transferase fusion protein (pGEX2TK vector, GE Healthcare Life Sciences, Little Chalfont, UK) containing residues 2-164 of dNdfip was purified and injected into rabbits by IMVS Veterinary Services Division (Adelaide, Australia). Anti-dNdfip was affinity purified and used at 1/100 for immunohistochemistry and $1 / 1000$ for immunoblot. Other primary antibodies used were: anti-Notch extracellular domain $(\mathrm{C} 458.2 \mathrm{H}$ ascites fluid; Developmental Studies Hybridoma Bank); anti-Notch intracellular domain (C17.9C6 ascites fluid; Developmental Studies Hybridoma Bank, lowa City, IA, USA); anti-cut (2B10, Developmental Studies Hybridoma Bank); mouse anti-wingless (4D4, Developmental Studies Hybridoma Bank); mouse anti-Rab5 and anti-Rab11 (BD-Transduction Laboratories, San Diego, CA, USA); mouse anti-HA (12CA5, Roche, Indianapolis, IN, USA); mouse anti-Flag (M2, Sigma, St Louis, MO, USA); rabbit anti-V5 (Bethyl Laboratories, Inc., Montgomery, TX, USA). Secondary antibodies used were Alexa-FLUOR 488- or 568-conjugated antisera (Invitrogen) and anti-Mouse-AP (Millipore, Billerica, MA, USA).

Immunohistochemistry and microscopy. At room temperature, SL2 cells were fixed for 10 min with $4 \%$ paraformaldehyde in PBS, then permeabilized with $0.2 \%$ TritonX-100 in PBS for 2 min, then blocked in 3\% FCS and 1\% BSA for $30 \mathrm{~min}$. Wing discs were fixed and stained as described previously. ${ }^{10}$ For live cell imaging, Lysotracker Red (Invitrogen) was applied as per manufacturer's instructions, then cells were washed in PBS, mounted and immediately analyzed. Confocal images were captured using a BioRad Radiance 2100 confocal microscope (Bio-Rad Microscience, Hertfordshire, UK; at Detmold Imaging Core Facility, Hanson Institute, Adelaide, SA, Australia) equipped with three lasers: Argon ion $488 \mathrm{~nm}$ (14 mw), Green HeNe $543 \mathrm{~nm}(1.5 \mathrm{mw})$ and Red Diode $637 \mathrm{~nm}(5 \mathrm{mw})$ and an Olympus IX70 inverted microscope. The objectives used were a 20x UAPOW (NA $=0.70)$ and a $60 x$ UPLAPO (NA = 1.2 water). The dual labelled cells were imaged with two separate channels (PMT tubes) in a sequential setting. Green fluorescence was excited with an Ar $488 \mathrm{~nm}$ laser line and the emission viewed through a HQ515/30 nm narrow band barrier filter in PMT1. Red fluorescence was excited with a HeNe $543 \mathrm{~nm}$ laser line and the emission viewed through a long pass barrier filter (E570LP) in PMT2. Automatically all signals from PMTs 1 and 2 were merged. Image analysis was performed with Confocal Assistant software for Microsoft Windows (Todd Clark Brelje, Bethesde, MD, USA).

\section{Conflict of interest}

The authors declare no conflict of interest.

Acknowledgements. We thank S Hayashi, M Baron and T Shandala for the provision of fly stocks, C Wesley for the stably expressing Notch cell line S2N and A Paterson for performing some of the real-time PCR analysis. We also thank the Bloomington and VDRC Stock Centers, the Australian Drosophila Biomedical Research Support Facility (OzDros) for fly lines, and Developmental Studies Hybridoma Bank (University of lowa) for antibodies. This work was funded by the National Health and Medical Research Council of Australia project grant (508085) and fellowship (399300) to SK.

1. Strizzi L, Hardy KM, Seftor EA, Costa FF, Kirschmann DA, Seftor RE et al. Development and cancer: at the crossroads of Nodal and Notch signaling. Cancer Res 2009; 69: $7131-7134$

2. Talora C, Campese AF, Bellavia D, Felli MP, Vacca A, Gulino A et al. Notch signaling and diseases: an evolutionary journey from a simple beginning to complex outcomes. Biochim Biophys Acta 2008; 1782: 489-497.

3. Wang Z, Li Y, Banerjee S, Sarkar FH. Emerging role of Notch in stem cells and cancer. Cancer Lett 2009; 279: 8-12.

4. Windler SL, Bilder D. Endocytic internalization routes required for delta/notch signaling. Curr Biol 2010; 20: 538-543

5. Wilkin M, Tongngok P, Gensch N, Clemence S, Motoki M, Yamada K et al. Drosophila HOPS and AP-3 complex genes are required for a Deltex-regulated activation of notch in the endosomal trafficking pathway. Dev Cell 2008; 15: 762-772.

6. Wilkin MB, Baron M. Endocytic regulation of Notch activation and down-regulation (review). Mol Membr Biol 2005; 22: 279-289.

7. Xu T, Artavanis-Tsakonas S. deltex, a Locus Interacting with the Neurogenic Genes, Notch, Delta and mastermind in Drosophila melanogaster. Genetics 1990; 126: 665-677. 
8. Hori K, Fostier M, Ito M, Fuwa TJ, Go MJ, Okano H et al. Drosophila deltex mediates suppressor of Hairless-independent and late-endosomal activation of Notch signaling. Development 2004; 131: 5527-5537.

9. Sakata T, Sakaguchi H, Tsuda L, Higashitani A, Aigaki T, Matsuno K et al. Drosophila Nedd4 regulates endocytosis of notch and suppresses its ligand-independent activation. Curr Biol 2004; 14: 2228-2236.

10. Wilkin MB, Carbery AM, Fostier M, Aslam H, Mazaleyrat SL, Higgs $\mathrm{J}$ et al. Regulation of notch endosomal sorting and signaling by Drosophila Nedd4 family proteins. Curr Biol 2004; 14: 2237-2244

11. Rotin D, Kumar S. Physiological functions of the HECT family of ubiquitin ligases. Nat Rev Mol Cell Biol 2009; 10: 398-409.

12. Yang B, Kumar S. Nedd4 and Nedd4-2: closely related ubiquitin-protein ligases with distinct physiological functions. Cell Death Differ 2010; 17: 68-77.

13. Shearwin-Whyatt L, Dalton $\mathrm{HE}$, Foot N, Kumar S. Regulation of functional diversity within the Nedd4 family by accessory and adaptor proteins. Bioessays 2006; 28: 617-628.

14. Jolliffe CN, Harvey KF, Haines BP, Parasivam G, Kumar S. Identification of multiple proteins expressed in murine embryos as binding partners for the WW domains of the ubiquitin-protein ligase Nedd4. Biochem J 2000; 351: 557-565.

15. Harvey KF, Shearwin-Whyatt LM, Fotia A, Parton RG, Kumar S. N4WBP5, a potentia target for ubiquitination by the Nedd4 family of proteins, is a novel Golgi-associated protein J Biol Chem 2002; 277: 9307-9317.

16. Konstas AA, Shearwin-Whyatt LM, Fotia AB, Degger B, Riccardi D, Cook DI et al. Regulation of the epithelial sodium channel by N4WBP5A, a novel Nedd4/Nedd4-2interacting protein. J Biol Chem 2002; 277: 29406-29416.

17. Shearwin-Whyatt LM, Brown DL, Wylie FG, Stow JL, Kumar S. N4WBP5A (Ndfip2) a Nedd4-interacting protein, localizes to multivesicular bodies and the Golgi, and has a potential role in protein trafficking. J Cell Sci 2004; 117: 3679-3689.

18. Oliver PM, Cao X, Worthen GS, Shi P, Briones N, Macleod M et al. Ndfip1 protein promotes the function of itch ubiquitin ligase to prevent $\mathrm{T}$ cell activation and T helper 2 cell-mediated inflammation. Immunity 2006; 25: 929-940.

19. Mund T, Pelham HR. Control of the activity of WW-HECT domain E3 ubiquitin ligases by NDFIP proteins. EMBO Reports 2009; 10: 501-507.

20. Foot NJ, Dalton HE, Shearwin-Whyatt LM, Dorstyn L, Tan S-S, Yang B et al. Regulation of the divalent metal ion transporter DMT1 and iron homeostasis by a ubiquitin-dependent mechanism involving Ndfips and WWP2. Blood 2008; 112: 4268-4275.

21. Howitt J, Putz U, Lackovic J, Doan A, Dorstyn L, Cheng H et al. Divalent metal transporter (DMT1) regulation by Ndfip1 prevents metal toxicity in human neurons. Proc Natl Acad Sci USA 2009; 106: 15489-15494.
22. Putz U, Howitt J, Lackovic J, Foot N, Kumar S, Silke J et al. Nedd4 family-interacting protein 1 (Ndfip1) is required for the exosomal secretion of Nedd4 family proteins. J Biol Chem 2008; 283: 32621-32627.

23. de Celis JF, Garcia-Bellido A, Bray SJ. Activation and function of Notch at the dorsalventral boundary of the wing imaginal disc. Development 1996; 122: 359-369.

24. Micchelli CA, Rulifson EJ, Blair SS. The function and regulation of cut expression on the wing margin of Drosophila: notch, Wingless and a dominant negative role for Delta and Serrate. Development 1997; 124: 1485-1495.

25. Bray SJ. Notch signaling: a simple pathway becomes complex. Nat Rev Mol Cell Biol2006 7: 678-689.

26. Jennings MD, Blankley RT, Baron M, Golovanov AP, Avis JM. Specificity and autoregulation of Notch binding by tandem WW domains in suppressor of Deltex. J Biol Chem 2007; 282: 29032-29042.

27. McGill MA, Dho SE, Weinmaster G, McGlade CJ. Numb regulates post-endocytic trafficking and degradation of Notch1. J Biol Chem 2009; 284: 26427-26438.

28. Chastagner $P$, Israel A, Brou C. AIP4/Itch regulates Notch receptor degradation in the absence of ligand. PLoS One 2008; 3: e2735.

29. Lieber T, Wesley CS, Alcamo E, Hassel B, Krane JF, Campos-Ortega JA et al. Single amino acid substitutions in EGF-like elements of Notch and Delta modify Drosophila development and affect cell adhesion in vitro. Neuron 1992; 9: 847-859.

30. Cellier M, Prive G, Belouchi A, Kwan T, Rodrigues V, Chia W et al. Nramp defines a family of membrane proteins. Proc Natl Acad Sci USA 1995; 92: 10089-10093.

31. Mund T, Pelham HR. Regulation of PTEN/Akt and MAP kinase signaling pathways by the ubiquitin ligase activators Ndfip1 and Ndfip2. Proc Natl Acad Sci USA 2010; 107 11429-11434.

32. Brand $\mathrm{AH}$, Perrimon N. Targeted gene expression as a means of altering cell fates and generating dominant phenotypes. Development 1993; 118: 401-415

33. Treier M, Staszewski LM, Bohmann D. Ubiquitin-dependent c-Jun degradation in vivo is mediated by the delta domain. Cell 1994; 78: 787-798.

34. Colussi PA, Quinn LM, Huang DC, Coombe M, Read SH, Richardson $\mathrm{H}$ et al. Debcl, a proapoptotic $\mathrm{Bcl}-2$ homologue, is a component of the Drosophila melanogaster cell death machinery. J Cell Biol 2000; 148: 703-714.

35. Cornell M, Evans DA, Mann R, Fostier M, Flasza M, Monthatong M et al. The Drosophila melanogaster Suppressor of deltex gene, a regulator of the Notch receptor signaling pathway, is an E3 class ubiquitin ligase. Genetics 1999; 152: 567-576.

36. Dietzl G, Chen D, Schnorrer F, Su KC, Barinova Y, Fellner M et al. A genome-wide transgenic RNAi library for conditional gene inactivation in Drosophila. Nature 2007; 448 151-156.

\section{Supplementary Information accompanies the paper on Cell Death and Differentiation website (http://www.nature.com/cdd)}

